\section{Sunspots and influenza}

SIR-Some years ago, Hope-Simpson ${ }^{1}$ pointed out a remarkable coincidence between peaks in the sunspot curve, when solar activity is at a maximum, and the occurrence of influenza pandemics associated with antigenic shifts of the virus. $\mathrm{He}$ pointed to five coincidences over the period 1919-68 as shown in the figure below. Using historical evidence on flu-like epidemics as summarized by Beveridge ${ }^{2}$, we extended the comparison before 1919 as shown ${ }^{3}$ :

$\begin{array}{cc}\text { Sunspot maximum } & \text { Date of pandemic } \\ 1761 & 1761-62 \\ 1767 & 1767 \\ 1778 & 1775-76 \\ & 1781-82 \\ 1787 & 1788-89 \\ 1804 & 1800-02 \\ 1830 & 1830-33 \\ 1837 & 1836-37 \\ 1848 & 1847-48 \\ 1860 & 1850-51 \\ 1870 & 1857-58 \\ 1893 & 1873-75 \\ & 1889-90\end{array}$

The first column ignores the abnormally low sunspot maxima in the years 1816 , 1883 and 1905, and the second column includes both "certain" and "possible" pandemics in the judgement of Beveridge $^{2}$. Although the correspondences between the figure and our table are clearly not precise, they do add credence to the speculation that solar activity and influenza activity may have a causal link. The two phenomena, which have irregular periods (with an average period of 11 years), appear to have kept in step over some 17 cycles.

Past experience has shown that false correlations of phenomena with the sunspot cycle may look good over a few cycles but go seriously adrift after an appreciable number of cycles. This does not happen for the postulated sunspot-flu connection.

Following the broad sunspot peak in 1968-70, the next peak occurred in 1979-80 coinciding closely with the 'Red Flu' pandemic of 1978-79 (see figure). The sunspot relative numbers from May to October 1989 are given as follows: 159 , $166,172,176,176,175$. The indications are that a peak has been reached in August/September 1989, and that this peak is the second highest on record. The highest recorded sunspot peak which occurred in 1957 coincided with the Asian flu pandemic of 1957-58. It is tempting to connect the recent flu epidemic in Britain with a maximum or imminent maximum of solar activity. The rise of sunspot numbers towards this peak is possibly amongst the steepest on record. Although the new wave of flu and flu-like illness has not yet assumed pandemic proportions, the chances of this happening within a period 1971-89. single complete cycle of terrestrial seasons must be reckoned to be high.

In conclusion we note that electrical fields associated with intense solar winds can rapidly drive charged particles of the size of viruses down through the exposed upper atmosphere into the shelter of the lower atmosphere, the charging of such particles being due to the photoelectric effect. This could define one possible

causal link between influenza pandemics and solar activity.

F. HOYLE

N. C. WICKRAMASINGHE

University of Wales

School of Mathematics,

Senghenydd Road,

Cardiff CF2 4AG, UK

1. Hope-Simpson, R.E. Nature 275, 86 (1978).

2. Beveridge, W.I.B. Influenza, the Last Great Plague (Heinemann, London, 1977).

3. Hoyle, F. \& Wickramasinghe, N.C. Diseases from Space (Dent, London, 1979)

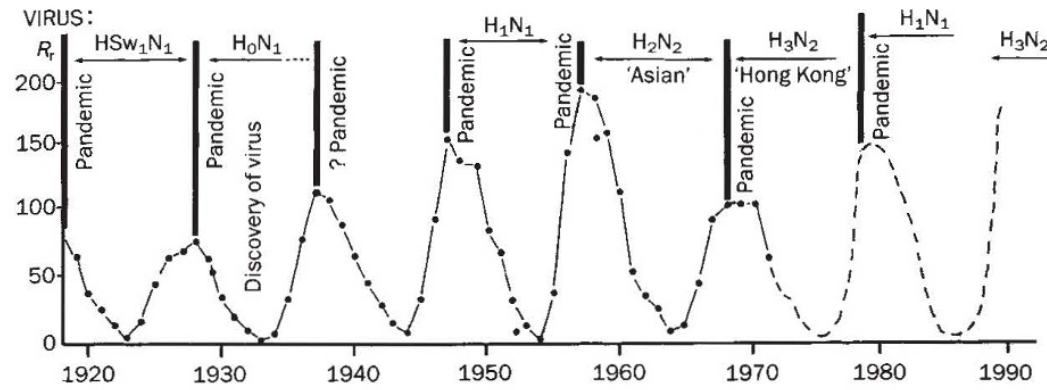

Yearly means of daily sunspot relative numbers compared with dates of influenza pandemics. The record up to 1971 is from Hope-Simpson; the dashed curve shows the situation for the

\section{Compound Q}

SIR-Robert Buderi in his article on the use of 'Compound Q' by AIDS patients in a programme organized by Project Inform (Nature 341, 247; 1989) added that a formal clinical trial of Compound Q was organized in May. We wish to emphasize that the materials undergoing evaluation in Project Inform's study and in a clinical trial approved by the Food and Drug Administration (FDA) are different, and that neither can accurately be described as Compound Q.

Trichosanthin is a protein isolated from the root tubers of Trichosanthes kirilowii, which has been used medically in China for a variety of clinical indications, chiefly induction of abortion. The name Compound $\mathrm{Q}$ originated as an informal, internal laboratory designation for trichosanthin during our initial collaborative in vitro studies of the material, at the University of California, San Francisco, and at Genelabs Incorporated, in Redwood City, California. Genelabs has developed a highly plurified, formulated form of trichosanthin designated GLQ223, now being produced by Genelabs according to FDA guidelines, which is the subject of an Investigational New Drug exemption granted by the FDA. Genelabs does not manufacture anything that can correctly be called Compound Q. There are no FDA-approved clinical studies of Compound Q currently being conducted at San Francisco General Hospital or anywhere else, nor are there any non-FDA approved studies being conducted with GLQ223.

The drug substance used by Project
Inform was a trichosanthin-containing preparation produced in the People's Republic of China, where the drug is used as an abortifacient. In the interest of avoiding confusion, we believe this material should be described as such, not through the use of the vague and imprecise term Compound $\mathrm{Q}$, which implies equivalence with GLQ223.

We believe that so far there are insufficient data to comment responsibly on either efficacy or toxicity of trichosanthin in HIV (human immunodeficiency virus) infected patients. As yet, neither the results from the FDA-approved trials of GLQ223 nor so far as we know, the results from Project Inform's activities, have clearly documented either a therapeutic benefit of trichosanthin treatment or the lack of such a benefit. On the basis of anecdotal information, some HIVinfected patients may potentially be predisposed toward serious clinical events, including the development of coma, following administration of trichosanthin. We believe that until additional information about the possible risks and benefits of administration of trichosanthin to HIV-infected patients is obtained, such administration should occur only under closely controlled circumstances and under the supervision of a physician, according to a protocol approved by the FDA.

Michael S. McGrath

San Francisco General Hospital, San Francisco, California 94110, USA JEFFREY D. LIFSON

Genelabs Incorporated, 505 Penobscot Drive,

Redwood City, California 94063, USA 